\title{
CORONAVIRUS ENVELOPE GLYCOPROTEIN ASSEMBLY COMPLEXES
}

\author{
Vinh-Phuc Nguyen' and Brenda G. Hogue ${ }^{1,2}$ \\ 'Division of Molecular Virology \\ ${ }^{2}$ Department of Microbiology and Immunology \\ Baylor College of Medicine \\ Houston, Texas 77030
}

\section{ABSTRACT}

Protein:protein interactions, and their subcellular localization, play important roles in coronavirus assembly. In this study, we have identified similar envelope glycoprotein complexes that are present in mouse hepatitis coronavirus A59 (MHV-A59) and bovine coronavirus (BCV) infected cells. Complexes consisting of the spike (S) and membrane (M) proteins were identified in cells infected with MHV-A59 or BCV. Kinetic analyses demonstrated that $\mathrm{S}$ and $\mathrm{M}$ quickly associated after translation, and suggested that both initially interacted in a pre-Golgi site. In addition, the hemagglutinin esterase (HE) was identified as part of a complex with $\mathrm{M}$ and $\mathrm{S}$ in BCV infected cells. Taken together, our data indicate that similar glycoprotein complexes are present in cells infected with two different coronaviruses, and thus likely represent important prerequisite complexes involved in virus assembly.

\section{INTRODUCTION}

All coronaviruses contain two major structural envelope glycoproteins, the membrane protein $(\mathrm{M})$ and the spike protein $(\mathrm{S})$. A few molecules of the envelope protein (E) are also found in purified virions. BCV is the prototype of coronavirus strains which, in addition to $\mathrm{S}$ and $\mathrm{M}$, also express a third major envelope glycoprotein, the hemagglutinin esterase (HE) (King, 1982). An interesting feature of the coronavirus life cycle is their assembly at the internal membranes (Tooze, 1984; Krijinse-Locker, 1994). This feature distinguishes them from other enveloped, RNA-containing viruses, like influenza virus and vesicular stomatitis virus which assemble and bud at the plasma membrane. In addition, 
coronaviruses do not possess a typical membrane-associated matrix protein. Thus, their assembly is thought to be mediated by direct interactions between the viral glycoproteins and the nucleocapsid at the membranes where virions bud. However, the precise molecular mechanism driving coronavirus assembly is not fully defined.

To investigate how the coronavirus glycoproteins are assembled into virions, we sought to identify protein complexes that are present in virus infected cells. Part of the data from our analyses of both MHV-A59 and BCV infected cells are presented here. Specific viral protein complexes that likely represent prerequisite components for the assembly of virions were detected by coimmunoprecipitations. Pulse-chase experiments were used to follow the kinetics of protein association in MHV-A59 infected cells. We then extended our study to include BCV since this virus expresses and incorporates $\mathrm{HE}$ into virions, in addition to $S$ and $M$. HE was identified in a complex with $M$ and $S$ in virus infected cells.

\section{MATERIALS AND METHODS}

\subsection{Viruses and Cell Lines}

MHV-A59 and BCV Mebus strains were grown in DBT and HCT-8 cells, respectively.

Both cell types were grown in Dulbecco's modified Eagle medium (Gibco BRL) supplemented with $10 \%$ fetal calf serum.

\subsection{Virus Infection, Radiolabeling, and Immunoprecipitation}

Cells were infected at a multiplicity of infection (moi) of 5-10. At $15 \mathrm{~h}$ post-infection with BCV or $5 \mathrm{~h}$ post-infection with MHV-A59, cells were starved for $30 \mathrm{~min}$ in methionine-deficient medium, labeled for $10 \mathrm{~min}$ with $200(\mathrm{Ci} / \mathrm{ml}$ of EXPRE35S35S (NEN), washed and harvested immediately or chased in the presence of excess methionine and cysteine for the indicated times.

Cells were lysed on ice in buffer containing $50 \mathrm{mM}$ Tris (pH 8.0), $62.5 \mathrm{mM}$ EDTA, $0.5 \%$ Nonidet P40 (NP40), $0.5 \%$ sodium deoxycholate (DOC) and $1 \mathrm{mM}$ phenylmethylsulfonyl fluoride (PMSF). Nuclei and cellular debris were removed by centrifugation at $4^{\circ} \mathrm{C}$ for $10 \mathrm{~min}$ at $13 \mathrm{~K} \mathrm{x} \mathrm{g}$. Clarified lysates were precleared with protein A Sepharose CL4B, and immunoprecipitated with specific antibodies. Antigen-antibody complexes were collected with protein A Sepharose and washed four times with RIPA buffer [ $50 \mathrm{mM}$ Tris$\mathrm{HCl}(\mathrm{pH} \mathrm{7.5)}, 150 \mathrm{mM} \mathrm{NaCl}, 1 \% \mathrm{NP} 40,0.5 \% \mathrm{DOC}, 0.1 \% \mathrm{SDS}$ ] and once in RIPA without detergents. Proteins were eluted in sample buffer, resolved on 5-20\% gradient gels by SDS-PAGE, and fluorographed.

\subsection{Antibodies}

All of the antibodies against the BCV proteins that were used in this study, with the exception of a polyclonal anti-BCV N, were previously described (Deregt and Babiuk, 1987; Deregt et al., 1987). The anti-BCV N polyclonal was made against histidine-tagged BCV N protein (Cologna and Hogue, unpublished data). The monoclonals against the MHV proteins were previously described (Fleming et al., 1983). 


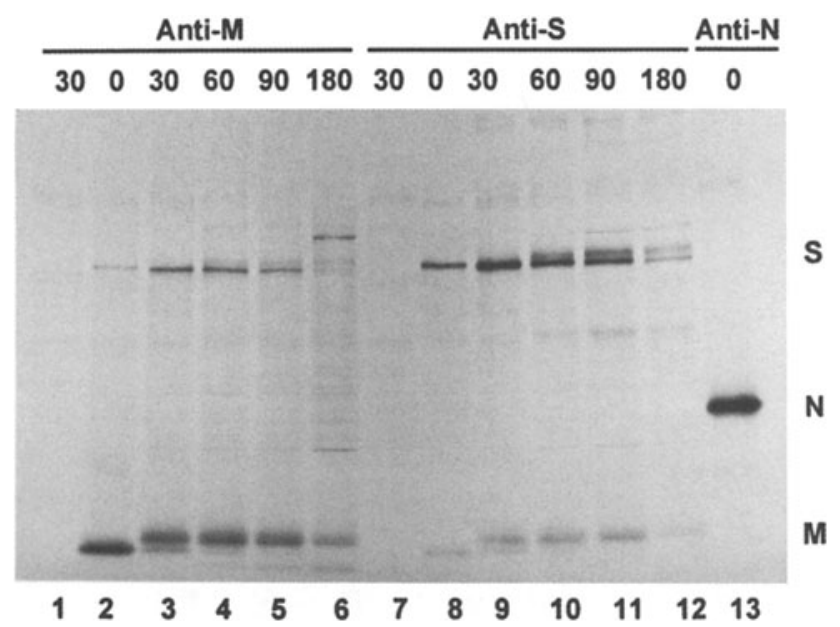

Figure 1. Kinetics of protein complex formation between MHV-A59 glycoproteins. At 5 hours post-infection, MHV-A59 infected DBT cells were pulsed with a mix of ${ }^{35} \mathrm{~S}$ methionine/cysteine for $10 \mathrm{~min}$, and chased in medium containing an excess of methionine and cysteine. At the indicated time points, cells were lysed, and immunoprecipitated with a mix of anti-M monoclonal antibodies (MAbs) (J1.3 and J2.7) (lanes 1-6), an anti-S MAb (J7.5) (lanes 7 to 12), or a mix of anti-N MAbs (J3.1 and J3.3) (lane 13). Eluted proteins were resolved by 5-20\% gradient SDS-PAGE, and fluorographed. Mock-infected cell lysates were used as negative controls (lanes 1 and 7). Letters denote the MHV-A59 protein designations: S (spike), $\mathrm{N}$ (nucleocapsid), and M (membrane).

\section{RESULTS}

To begin our studies on virus assembly we initially identified protein complexes that were present in MHV-A59 infected cells. Mock- and virus infected cells were pulse labeled for 10 minutes with ${ }^{35} \mathrm{~S}$ methionine/cysteine and chased in the presence of excess label. Complexes consisting of $\mathrm{S}$ and $\mathrm{M}$ were detected immediately after the pulse when lysates were immunoprecipitated with either anti-M or anti-S antibodies (Fig. 1, lanes 2 and 8). The MHV-A59 $\mathrm{M}$ has been shown to be unglycosylated in the endoplasmic reticulum (ER), and becomes O-glycosylated in the Golgi (Krijinse-Locker, 1992). After a 10 min of pulse, the S-associated $\mathrm{M}$ appeared to be unglycosylated, and subsequently acquired sugar chains through the chase (Fig. 1, compare lane 12 to 8). Similar post-translation modifications, namely $\mathrm{N}$-linked glycosylation, also appeared to occur on the $\mathrm{M}$-associated S (Fig. 1, compare lane 6 to 2). This suggests that $\mathrm{S}$ and $\mathrm{M}$ initially associated in a pre-Golgi site, most likely in the ER. The S-M complexes were subsequently transported along the exocytic pathway, probably as part of assembled virions.

Next we extended our studies on coronavirus assembly to include BCV, with an interest in the hemagglutinin esterase (HE) glycoprotein. To date, the molecular mechanism of incorporation of $\mathrm{HE}$ into virions has not been addressed. HCT cells were infected with BCV Mebus strain at a moi of 10 . At $15 \mathrm{~h}$ post-infection, virus infected cells were metabolically labeled for $20 \mathrm{~min}$, chased for 1 hour, lysed and immunoprecipitated with specific anti-BCV antibodies. Similar to MHV-A59 infected cells, complexes consisting of S and M were also identified (Fig. 2, lanes 3-4). Furthermore, HE was also found in a complex with S-M (Fig. 2, 


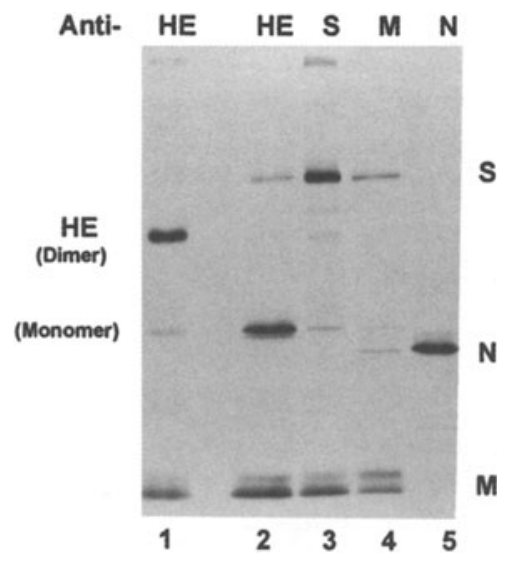

Figure 2. Detection of protein complexes in $\mathrm{BCV}$ infected cells. At 15 hours post-infection, BCV infected HCT cells were metabolically pulsed for $20 \mathrm{~min}$, chased for $1 \mathrm{~h}$, lysed and immunoprecipitated wit $h$ specific anti-BCV protein antibodies. To visualize HE dimers, immunoprecipitated proteins were eluted in sample buffer without $\beta$-mercaptoethanol (nonreducing conditions) (lane 1 ).

lane 2-4). When proteins were eluted in the absence of reducing agent, the majority of immunoprecipitated HE was the dimeric form (Fig. 2, lane 1). Parallel immunoprecipitation with a rabbit anti-N antiserum showed that $\mathrm{HE}$ and $\mathrm{S}$ are not in a complex with $\mathrm{N}$, even though a small amount of N-M complexes were coimmunoprecipitated (Fig. 2, compare lane 4-5).

\section{DISCUSSION}

Both protein:protein and protein:RNA interactions, as well as the subcellular localization of protein complexes, are thought to be important for coronavirus assembly. To date, the molecular mechanism of coronavirus assembly remains largely undefined. This study aimed at examining the interactions between the viral envelope proteins that ultimately lead to the formation of mature particles in virus infected cells. In this report, we showed that similar protein complexes were detected by coimmunoprecipitation from cells infected with either MHV-A59 or BCV. The complexes are likely important prerequisite components for assemby of virions.

Our data showed that in MHV-A59 infected cells, complexes of S and M were readily detected after a $10 \mathrm{~min}$ pulse. This suggests that these glycoproteins quickly associate after synthesis. Shorter pulse-labeling yielded identical results (data not shown). The kinetics of M-S complex formation differ from those previously reported for MHV (Opstelten et al., 1995). Different cells and antibodies may account for this. Otherwise, our data are consistent with the previously reported results.

The $\mathrm{M}$ glycoprotein has been shown to be transported to the Golgi when it is expressed in the absence of other coronavirus proteins (Klumperman, 1994). Nevertheless, it remains the only viral protein that is retained in the same general area where coronaviruses bud. Therefore it has been proposed that $\mathrm{M}$, through self-oligomerization and interactions with other viral proteins, retains and organizes the glycoproteins in the membrane so the ribonucleocapsid complex can bind and initiate virion budding (KrijinseLocker, 1995). Based on their glycosylation patterns, S and M appear to initially associate at a pre-Golgi site, presumably in ER, and the formed complexes are subsequently transported to the Golgi. Our results correlate well with the proposed scheme of events that lead to the formation of coronavirus particles. 
The HE glycoprotein is a major BCV envelope glycoprotein that is absent from MHV A59 virions (Hogue, 1984). To date, the molecular mechanism of $\mathrm{HE}$ incorporation in $\mathrm{BCV}$ particles has yet to be addressed. This is the first report describing the presence of HE in a complex with $\mathrm{S}$ and $\mathrm{M}$. HE does not directly interact with $\mathrm{S}$, but initially interacts with $\mathrm{M}$ and later becomes part of complex consisting of S-M-HE (Nguyen and Hogue, submitted). HE has been shown to rapidly dimerize after synthesis (Hogue, 1989). It is unknown at present which form of HE, monomeric or dimeric, initially associates with $\mathrm{M}$; however, only dimers are found as part of S-M-HE complexes (Nguyen and Hogue, submitted).

Taken altogether, our data showed that the viral envelope glycoproteins associate to form stable complexes. These complexes are present in both MHV and BCV infected cells. Since common complexes are detected in cells infected with two different coronaviruses, this supports the idea that these are important preassembly complexes. An additional glycoprotein, HE, is readily accommodated as part of the glycoprotein complexes. These complexes presumably create a favorable micro-environment which excludes cellular proteins, and allows the nucleocapsid to bind and form budding progeny virions.

\section{ACKNOWLEDGMENTS}

We thank John Fleming and Lorne Babiuk for generously providing antibodies. This work was supported by Public Health Service grant AI33500 from the National Institutes of Health.

\section{REFERENCES}

Deregt, D. and L. A. Babiuk., 1987, Monoclonal antibodies to bovine coronavirus: characteristics and topographical mapping of neutralizing epitopes on E2 and E3 glycoproteins, Virology 161:410-420.

Deregt, D., M. Sabara, and L. A. Babiuk, 1987, Structural proteins of bovine coronavirus and their intracellular processing, J. Gen. Virol. 68:2863-2877.

Fleming, J. O., S. A. Stohlman, R. C. Harmon, M. M. Lai, J. A. Frelinger, L. P. Weiner., 1983, Antigenic relationships of murine coronaviruses: analysis using monoclonal antibodies to JHM (MHV-4) virus, Virology 131:296-307

Hogue, B. G., B. King, and D. A. Brian., 1984, Antigenic relationship among proteins of bovine coronavirus, human respiratory coronavirus OC43, and mouse hepatitis coronavirus A59, J. Virol. 51:384-388.

Hogue, B. G., T. E. Kienzle, and D. A. Brian., 1989, Synthesis and processing of the bovine enteric hemagglutinin protein, J. Gen. Virol. 70:345-352.

King, B., and D. A. Brian., 1982, Bovine coronavirus structural proteins, J. Virol. 42:700-707.

Klumperman, J., J. Krijinse-Locker, A. Meijer, M. C. Horzinek, H. J. Geuze, and P. J. M. Rottier., 1994, Coronavirus $\mathrm{M}$ proteins accumulate in the Golgi complex beyond the site of virus budding, J. Virol. 68:6523-6534.

Krijinse-Locker, J., G. Griffiths, M. C. Horzinek, and P. J. M. Rottier., 1992, O-glycosylation of the coronavirus M protein, J. Biol. Chem. 267:14094-14101.

Krijinse-Locker, J., M. Ericsson, P. J. M. Rottier, and G. Griffiths., 1994, Characterization of the budding compartment of mouse hepatitis virus: Evidence that transport from the RER to the Golgi complex requires only one vesicular transport step, J. Cell. Biol. 124:55-70.

Krijinse-Locker, J., D.-J. E. Opstelten, M. Ericsson, M. C. Horzinek, and P. J. M. Rottier., 1995, Oligomerization of a trans-Golgi/trans-Golgi network retained protein occurs in the Golgi complex and may be part of its retention, J. Biol. Chem. 270:8815-8821.

Opstelten, D.-J. E., M. J. B. Raasmsman, K. Wolfs, M. C. Horzinek, and P. J. M. Rottier., 1995, Envelope glycoprotein interactions in coronavirus assembly, J. Cell. Biol. 131:339-349.

Tooze, J., S. A. Tooze, and G. Warren., 1984, Replication of coronavirus MHV-A59 in Sac- cells: determination of the first site of budding of progeny virions, Eur. J. Cell. Biol. 33:281-293. 